\title{
High Spatial Resolution Measurement of Low Magnetic Fields by Needle-Type GMR Sensor
}

\author{
H. Hoang, R. Haraszczuk, M. Kakikawa, T. Ueno, S. Yamada \\ Institute of Nature and Environmental Technology, Kanazawa Univ., Kakuma-machi, Kanazawa, Ishikawa 920-1192, Japan
}

In this paper, we propose a measurement of a nerve signal model and a printed circuit for low-level and high-density signals as examples of low magnetic field applications that require minimal invasive measurement or operations in narrow space. Fabricated GMR sensor, with sensing element of high sensitivity at the tip of the needle, can be placed much closer to the source of the magnetic field. Extremely small size and specific construction of this needle type sensor indicates that it is an excellent device for above mentioned applications.

Key words: GMR sensor, needle structure, spatial resolution, sensitivity, low-level, high distribution

\section{Introduction}

Detection and measurement of low level local magnetic fields has been studied for years in medical as well as industrial applications. The relatively large and cumbersome magnetometers are used there for monitoring magnetic signals. However, these kinds of sensors need to be placed at a specific distance, a little bit far away from the target objects, that results in general signals of a wide area. For extremely small dimension of measured objects, sensing distance is required to be as small as possible (much less than 1 $\mathrm{mm}$ ). Therefore, miniature sensing element with high sensitivity is required. The principle of this research is to point out that GMR needle type sensor (which has extremely small dimensions) can be used to examine magnetic fields coming from area smaller than $1 \mathrm{~mm}^{2}$ in the near future.

\section{Low level local magnetic field detection}

\subsection{Measurement objects}

As it is well known, once the nerve is stimulated it introduces an electrical signal and this is propagated along the nerve axon. Many research from early days on the nerve signal discovered that when the nerve is not stimulated, it possesses a negative resting membrane potential (about $-70 \mathrm{mV}$ in human body). As the nerve signal is generated, the chain-reaction of protein ions along axon occurs through the protein channels ${ }^{1}$. This is due to the rushed movements of the protein ions between outside and inside neuron, such as sodium $\mathrm{Na}^{+}$ and potassium $\mathrm{K}^{+}$. The imbalance of the protein ions around the nerve axon leads to the action potential, which consists of the processes called depolarization and repolarization. These two phases can be assumed as two current dipoles in opposite directions flowing in nerve axon $^{2,3)}$, as it is shown in Fig. 2(a).

Therefore, taking the action potential principle into account, we have developed a model of nerve signal propagating along axon as a chain of opposite current dipoles, which generates the magnetic field. This field is detected by the novel needle-type GMR sensor. Action current propagation was modeled by a pulse signal with $20 \%$ duty cycle at a frequency of $1 \mathrm{kHz}$. This signal was supplied to a wire of $20 \mu \mathrm{m}$ diameter which was knitted into holes of $200 \mu \mathrm{m}$ diameter drilled in a silicon plate with a fixed distance of $1 \mathrm{~mm}$ as depicted in Fig. 2(b).

Additionally, a dense three-roll mesh coil printed on the circuit board with dimensions of about $1 \mathrm{~cm} \times 1 \mathrm{~cm}$, as shown in Fig. 5, is suggested as another measurement object for low level local magnetic field detectability. The diameter of the coil is approximate $100 \mu \mathrm{m}$ and the distance between coils is $100 \mu \mathrm{m}$ as well.

For this low level local magnetic field detection, distance between the sensing element and the measured objects is required to be as small as possible (much less than $1 \mathrm{~mm}$ ). That results in a possibility of giving a larger output signal ${ }^{4)}$. Thus, needle-type GMR sensor with distinguished characteristic, as described below, is proposed for this measurement.

\subsection{Advantages of GMR needle probe}

The needle-type GMR sensor probe consists of especially designed needle. The needle is fabricated from a compound of Aluminum Oxide and Titanium Carbide $\left(\mathrm{Al}_{2} \mathrm{O}_{3} / \mathrm{TiC}\right)$. Moreover, it is only $20 \mathrm{~mm}$ in length and hundreds of $\mu \mathrm{m}$ in width. Therefore, it could measure inside body with a minimal invasion. GMR sensing element with both small size in the order of micron (75 $\mu \mathrm{m} \times 40 \mu \mathrm{m})$ and high sensitivity of $18 \mu \mathrm{V} / \mu \mathrm{T}$ was placed at the tip of the needle as it is shown in Fig. 1. In consequence, this type of probe is expected to be able to detect a low current signal locally with distance of 0.01 $\mathrm{mm}$ order as well as high spatial resolution of $0.1 \mathrm{~mm}$ order. Input voltage supplied to the sensor in these experiments was about $\pm 9 \mathrm{~V}$.

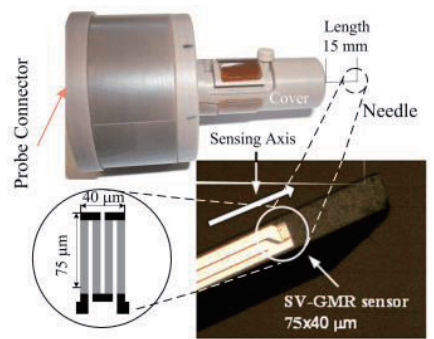

Fig. 1 Needle-type GMR sensor. 


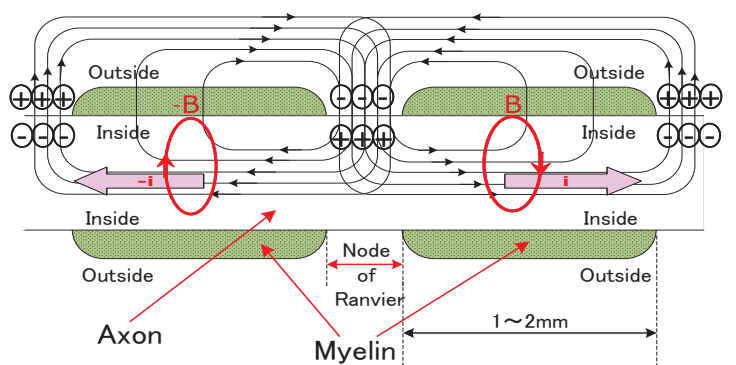

(a) Principle of action current propagation

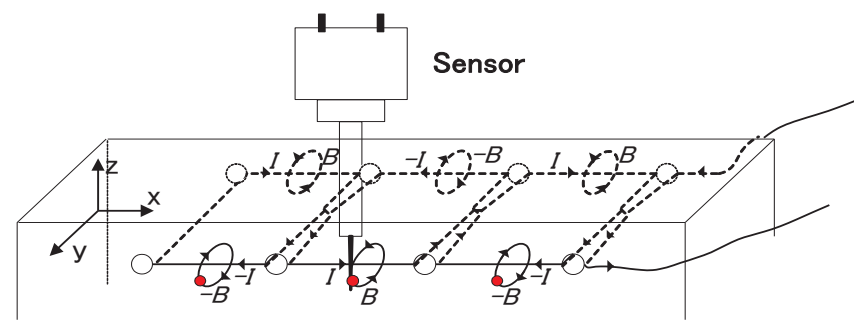

(b) Model of nerve signal

Fig. 2 Action potential of nerve.

\section{Experimental setup and measurement method}

\subsection{Experimental setup}

Considering the fact that for low frequencies from $10^{-1}$ to $10^{4} \mathrm{~Hz}$ the intrinsic noise of GMR sensor, normally due to its multilayer structure, is on the order of $10^{-10}$ $\mathrm{T} / \mathrm{Hz}^{1 / 2}$ ) , we have devised an experimental setup as it is shown in Fig. 3.

The low magnetic field source is produced by a considerably ideal low current source, which is supplied by a multifunction synthesizer (WF 1965 1CH) connected to the variable high resistors, in order to reduce unwanted noise from power supply. The resistors of $100 \mathrm{k} \Omega \sim 1 \mathrm{M} \Omega$ were exploited providing a stable low current in the order of several $\mu \mathrm{A}$ to hundreds of $\mu \mathrm{A}$. Output signal obtained by GMR sensor was amplified 10000 times using the amplifier AD524 of a very low noise. After that we forwarded it to either the digital oscilloscope (DL4100 Yokogawa), which averaged the experimental data at about 2048 times, or the lock-in amplifier (NF Electronics, LI5640).

Moreover, the sensor probe is designed as a bridge circuit structure consisted of four GMR sensing elements, where one is placed on the tip of the needle and three others are near the bonding pad. It is aimed to decrease the noise in the measurement.

\subsection{Measurement method}

In the experiments, sensor was fixed perpendicularly to the model at a specified distance in $y$ direction (approximately $20 \mu \mathrm{m}$ ), while the model was moved along the horizontal $x$ axis as shown in Figs. 4 and 5 .

Distance in $y$ direction is measured by thin polyimide foils with different thicknesses. For all experiments, distance of approximately $20 \mu \mathrm{m}$ was constant in $y$ direction. The polyimide layer of $20 \mu \mathrm{m}$ thickness was placed between the measured object and the sensor, which were fixed in $x$ and $z$ direction. Then the measured object was being moved toward the sensor until it touched the polyimide layer. The thickness of the layer is expected to state the distance between the target object and the sensor in $y$ direction. Due to check, the identical layer of $30 \mu \mathrm{m}$ thickness was employed, as the space of $20 \mu \mathrm{m}$ is supposed not to let the layer of $30 \mu \mathrm{m}$ thickness go through.

Even though micrometers were utilized to identify the distance in each direction during the experiment, additionally the position of the sensor was checked by using magnifying glass. However, it should be noticed that the positioning error of several $\mu \mathrm{m}$ (much less than $10 \mu \mathrm{m})$ has possibly occurred.

Next, to verify whether the model is set in the position consistent with the measurement coordinate system, we input signal of comparably big current of $100 \mu \mathrm{A}$, which produces output signal possible to be observed clearly from the oscilloscope. Since the signals at points y01 and yo2 in Fig. 4 are supposed to be nearly the same, we keep on adjusting the screws in the measurement system until the same signals at those two points are caught.

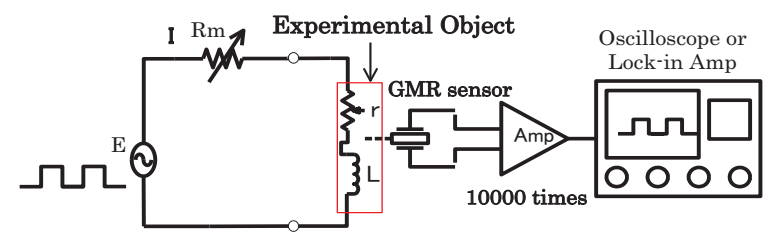

Fig. 3 Equivalent circuit of experimental setup.

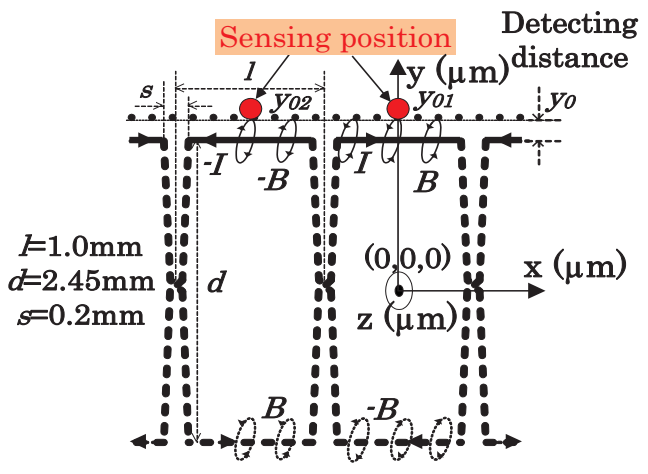

Fig. 4 Model of nerve signal.

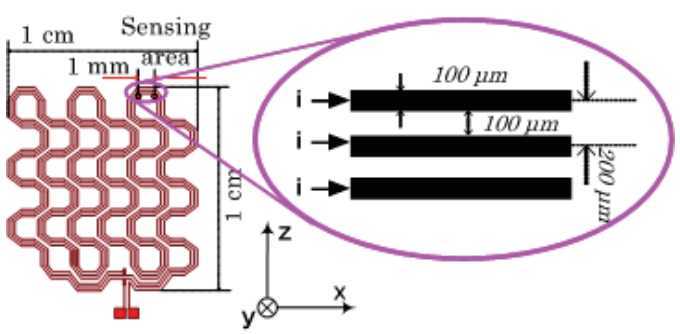

a) Mesh coil

b) Paralell coils

Fig. 5 Mesh coil as high density circuit. 


\section{Experimental results}

\subsection{Theoretical results of magnetic flux density $\mathrm{Bz}$ of the} nerve model

Theoretical values of the magnetic flux density $B Z$ in experimental model can be calculated by the following equation, derived from Law of Bio-Savart:

$$
d \vec{B}=\frac{\mu i}{4 \pi} \frac{d \vec{l} \times \vec{r}}{\mathrm{r}^{3}}
$$

where the vector $d B$ is a magnetic flux density, the product vector of $i$ and vector $d l$ is current element, and vector $r$ is the distance from the center axis of wire.

Fig. 6 shows the analysis result of magnetic field distribution of the nerve model. As it is easily seen, the magnetic flux density is zero at the middle point in $x$ axis, which is considered to correspond to the holes in our nerve model because of flux cancelling to each other. Along two other sides from this point in $x$ axis, the magnetic flux density increases in absolute values and reaches the peak at the points 0 and $1 \mathrm{~mm}$ in $x$ direction.

\subsection{Experimental results from the nerve signal}

Pulse signal of $5 \mu \mathrm{A}$ amplitude modulated with $20 \%$ duty cycle at $1 \mathrm{kHz}$ frequency was input. The nerve signal model was placed perpendicularly to the sensor, fixed in $y$ and $z$ direction, and moved along horizontal $x$ axis in $100 \mu \mathrm{m}$ steps. The output signal from the sensor was 10000 times amplified before being observed by a digital oscilloscope with the averaging of 2048 times, then the data was transferred to the computer by Matlab program.

Fig. 7 indicates that the spatial distribution of magnetic flux density (vertical axis) of the nerve model depends on the distance in $x$ direction (horizontal axis) what resulted of the sensor measurements. The magnetic flux density $B Z_{p p}$ of approximate $30 \mathrm{nT}$ was detected with the pulse input current of $5 \mu \mathrm{A}$ in a spatial resolution of about $100 \mu \mathrm{m}$. The magnetic flux density indicated in vertical axis of Fig. 7 was obtained by dividing the sensor's output voltage by a gain of 180000 corresponding to the sensitivity of the sensor $(18 \mu \mathrm{V} /$ $\mu \mathrm{T})$ before 10000 times amplification. The described results show that the experimental data agrees quite well with theoretical calculation, only varying among some $\mu \mathrm{m}$ in distance from the sensor to the model.

\subsection{Experimental results from the mesh coil}

Low current of $10 \mu \mathrm{A}$ or $5 \mu \mathrm{A}$ at frequency of $1 \mathrm{kHz}$ was supplied while the dense mesh coil was being moved along $x$ axis. Taking into consideration that dimension of the sensing element is $75 \mu \mathrm{m} \times 40 \mu \mathrm{m}$, scanning steps of $100 \mu \mathrm{m}$ were chosen. The magnetic flux density distribution of output signal from the mesh coil due to position change in $x$ and $z$ direction is depicted in Figs. 8, 9. The GMR sensor output was measured by a digital lock-in amplifier after being 10000 times amplified. As it is indicated in Fig. 9(a) a higher current of $100 \mu \mathrm{A}$ with $50 \mu \mathrm{m}$ steps was input to observe the variation of magnetic flux density achieved from the sensor. Base on the result obtained in Fig. 9(a), low current of $10 \mu \mathrm{A}$ or $5 \mu \mathrm{A}$ was applied. Fig. 9(b) showed that even with a spatial resolution of $100 \mu \mathrm{m}$ the variation of magnetic flux density can be observed with similar tendency to that in Fig. 9(a).

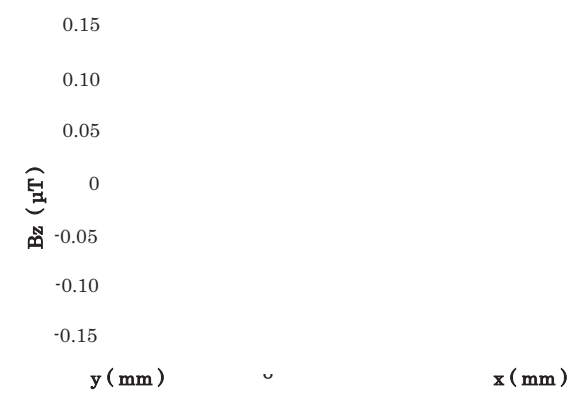

Fig. 6 Magnetic flux density distribution analysis.

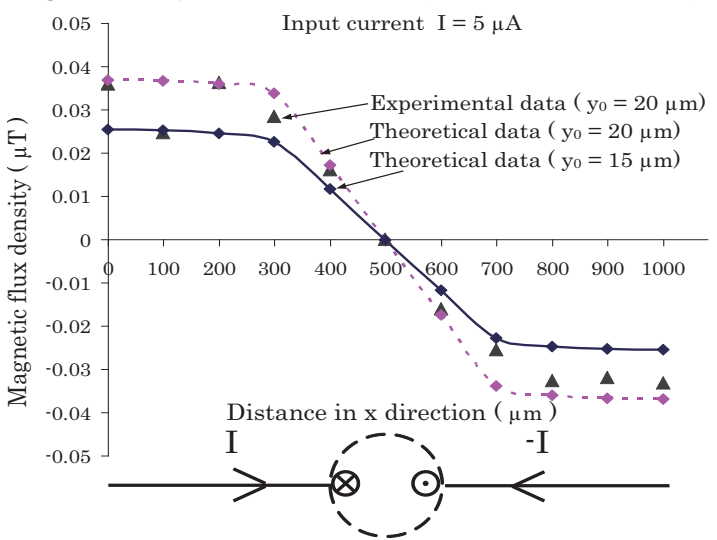

Fig. 7 Spatial distribution of magnetic flux density.

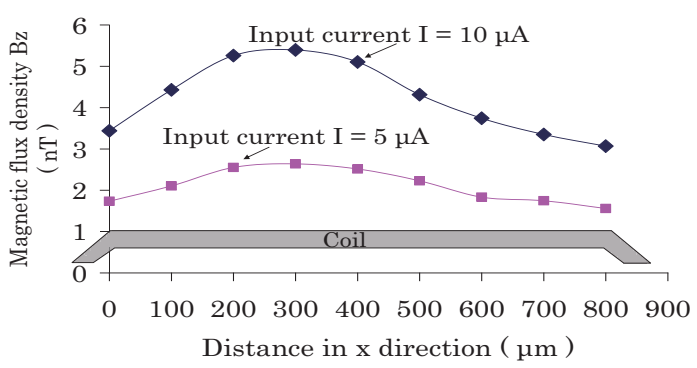

Fig. 8 Dependence of magnetic flux density $B z$ of output signal on distance in $\mathrm{x}$ direction.

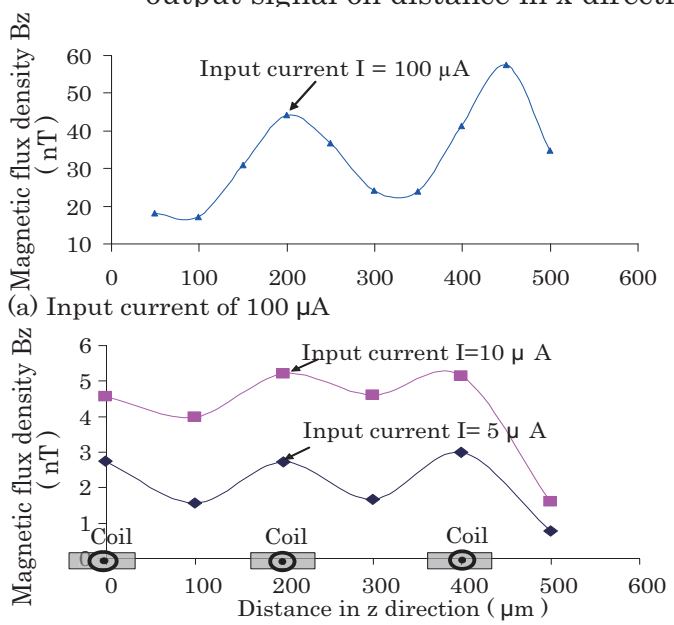

(b) Input current of $10 \mu \mathrm{A}$ and $5 \mu \mathrm{A}$

Fig. 9 Dependence of magnetic flux density $B Z$ of output signal on distance in $\mathrm{z}$ direction. 


\subsection{Characteristic of detected signal}

Giving as an example, the output signal waveform detected by the sensor is presented in Fig. 10(b), which is in phase with input signal which amplitude is $10 \mu \mathrm{A}$ (see Fig. 10(a)). Furthermore, Figs. 11, 12, 13, and 14 give the detected signals which are either independent of frequency of input signal or proportional to the input amplitude. The Figs. 11 and 13 show the change of the output signal detected by a sensor, which was 10000 times amplified and obtained by lock-in amplifier, is dependent on amplitude of input current changing in the range of $5 \mu \mathrm{A} \sim 100 \mu \mathrm{A}$. Figs. 12 and 14 present the output voltage obtained with 10000 times amplification up to the change of the frequency of input signal from $100 \mathrm{~Hz} \sim 3000 \mathrm{~Hz}$.

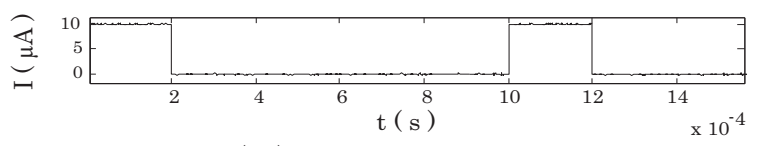

(a ) Input signal waveform

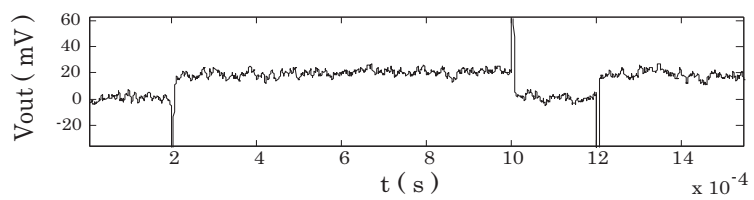

( b ) Output signal waveform

Fig. 10 Comparison of output and input signal.

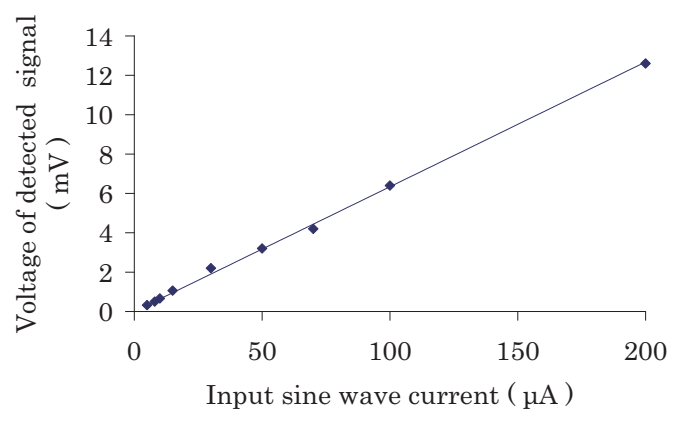

Fig. 11 Dependence of output voltage on input current of the nerve model.

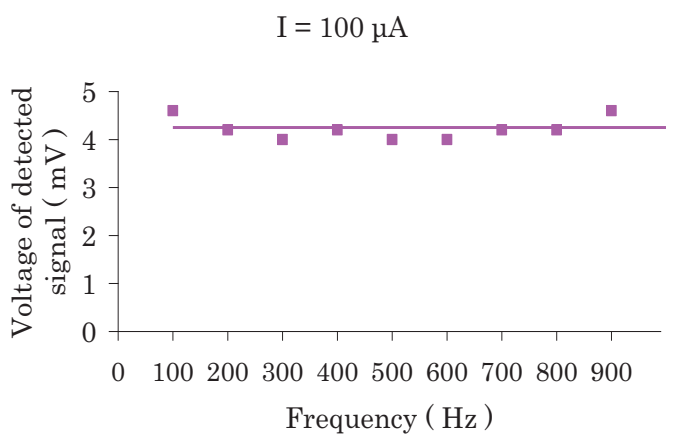

Fig. 12 Frequency characteristic of the nerve model.

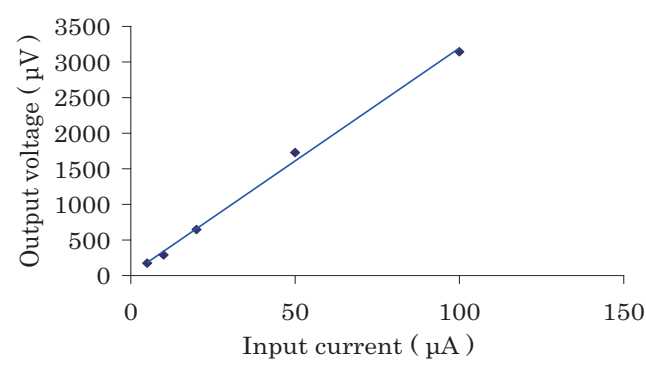

Fig. 13 Dependence of output voltage on input current of the mesh coil.

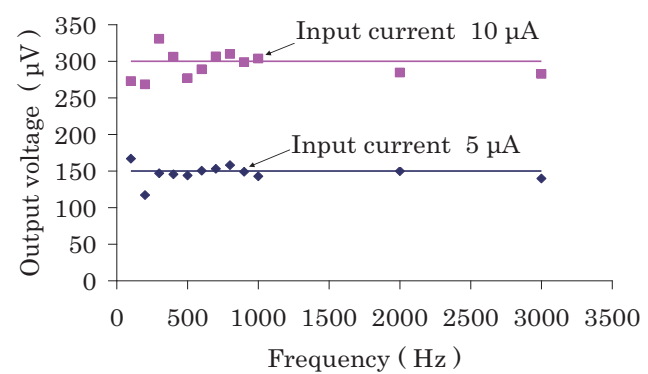

Fig. 14 Frequency characteristic of the mesh coil.

\section{Conclusion}

Experimental results indicate a possibility of detecting the magnetic field generated by current signals as low as $5 \mu \mathrm{A}$ by the novel needle-type GMR sensor probe. There is a possibility to detect low level local magnetic field, due to the specific structure, high sensitivity and spatial resolution up to about $100 \mu \mathrm{m}$ of this GMR sensor probe. Moreover, the detected signals were comparable with the expected theoretically calculated characteristics. Nevertheless, the output signals are in phase with input ones as well as are independent of the change in frequency and are proportional to the amplitude of input signals. The experiments were performed in the shielding room as well as lock-in amplifier was used to prevent undesirable signals. In the near future, we would like to improve our experimental setup as well as experimental technique (such as sensing position variation) to decrease the unexpected noise to find out the possibility of detecting as low current signal with high resolution as possible.

\section{References}

1) E.R.Kandel, J.H.Schwartz, T.M.Jessell, Principles of Neural Science, 3rd ed. (Appleton\&Lange), pp. 23-25, pp.101-105.

2) S.J.Williamson, M.Hoke, G.Stroink, M.Kotani, Eds., Advances in Biomagnetism (Plenum, N.Y, 1989), pp.1-18.

3) J.P.Wikswo, J.P.Barach, J. A. Freeman, Science, 208 (4439), 53 (1980).

4) A. Lekawa, C.P. Gooneratne, K.Chomsuwan, M.Iwahara, H.Stryczewska, S.Yamada, J. of Japan Society of Applied Electromagnetics and Mechanism, 16, 3, 221 (2008).

5) N.A.Stutzke, S.E.Russek, D.P.Pappas, J. Appl. Phys. 97, 10Q107 (2005).

Received Apr. 20, 2010; Revised May. 10, 2010; Accepted May. 20,2010 